\title{
ROBUST CONTROL OF AN ELECTROHYDRAULIC SERVO SYSTEM UTILIZING ONLINE ESTIMATE OF ITS NATURAL FREQUENCY
}

\author{
Hideki YANADA* and Kazumasa FURUTA** \\ * Department of Mechanical Engineering, Faculty of Engineering \\ Toyohashi University of Technology \\ 1-1, Hibarigaoka, Tempaku-cho, Toyohashi, 441-8580 Japan \\ (E-mail: yanada@mech.tut.ac.jp) \\ ** Harmonic Drive Systems, Inc. \\ 1856-1, Oaza-maki, Hotaka-machi, Minamiazumi-gun, 399-8305 Japan
}

\begin{abstract}
This paper deals with online parameter estimation of an electrohydraulic servo (EHS) system and with robust control of the EHS system utilizing the parameter estimated online. In this study, the natural frequency of the EHS system is estimated using pressure sensors based on the equation of motion and equation of continuity, and the estimate of the natural frequency is utilized to enhance the robustness of the EHS system combined with a parallel feed-forward compensator (PFC). The optimal value of one of the PFC parameters is strongly influenced by the natural frequency of the EHS system and is adaptively varied using the estimate of the natural frequency. It is shown that the natural frequency of the EHS system can be estimated relatively accurately using the method proposed, and that the robustness of the EHS system to the plant parameter variations can be enhanced by adapting the PFC parameter.
\end{abstract}

\section{KEY WORDS}

Electrohydraulic servo system, Robust control, Parameter estimation, Parallel feedforward compensator

\section{NOMENCLATURE}

$A_{\mathrm{i}}:$ piston area $(\mathrm{i}=1,2)$

$B_{e}$; effective bulk modulus of fluid

$e_{y}:$ error $\left(=y_{p}-y_{m}\right)$

$F_{L}$ : external force plus frictional force of cylinder

$J_{e}:$ evaluation function

$K$ : gain of EHS system

$k_{e}:$ proportional gain

$m$ : load mass

$p_{\mathrm{i}}:$ pressure $(\mathrm{i}=1,2)$

$p_{s}:$ supply pressure

$q_{\mathrm{i}}:$ flow rate $(\mathrm{i}=1,2)$

$r$ : reference input

$s$ : Laplace variable or differential operator $t \quad$ : time

$u_{p}:$ control input (servo current)

$V_{\text {i }}: \quad$ volume of fluid under compression $(\mathrm{i}=1,2)$

$v:$ piston velocity

$x_{s}:$ spool displacement

$y_{a}:$ output of augmented plant

$y_{m}:$ output of reference model

$y_{f}:$ output of PFC

$y_{p}:$ piston displacement

$\alpha:$ PFC parameter

$\beta$ : PFC parameter

$\zeta$ : damping ratio of EHS system

$\lambda_{h}:$ break frequency of highpass filter

$\lambda_{l}:$ break frequency of lowpass filter

$\omega_{n}:$ natural angular frequency of EHS system 


\section{INTRODUCTION}

An electrohydraulic servo (EHS) system usually suffers from parametric variations and disturbances. When the magnitudes of the parametric variations and/or the disturbances are large, the control performance of the EHS system degrades to some or a great degree even in the case where a robust control scheme such as sliding mode control or $\mathrm{H}$ infinity control is used. In such a case, an adaptive algorithm needs to be incorporated. This paper treats an online estimation of the natural frequency of the EHS system, which is the most important of the linearization parameters, as well as the update of the controller parameters using the estimate of the natural frequency.

Simple adaptive control (SAC) theory is considered to be one of the most promising control theories due to its simple structure and high robustness [1-3]. In order for the SAC scheme to be applied, a plant must be almost strictly positive real (ASPR). Most plants including EHS systems, however, do not satisfy the ASPR condition. In such a case, a feedforward compensator is added in parallel with the plant, and the compensator is called the parallel feedforward compensator (PFC). The PFC is added in order for the augmented plant, which is the parallel combination of the plant and the PFC, to satisfy the ASPR conditions [1].

Some methods to determine the structure of the PFC have so far been proposed [1], but no clear method to determine the parameters included in the PFC has been proposed to date. Because of this, the PFC parameters are usually determined by trial and error. The PFC parameters have a strong influence on both the ASPR conditions and the response characteristic of the plant output. In addition, the optimal values of the PFC parameters are strongly dependent on the plant parameters. Therefore, the control performance may degrade when the plant parameters are largely varied, even though the ASPR conditions are maintained.

This investigation aims at developing a robust controller by which a good performance is always attained even under large variations of the EHS system's parameters. In this paper, a method to tune the PFC parameters online, which is based on the value of the natural frequency of the EHS system estimated online, is proposed and the usefulness of the scheme is examined. The organization of the paper is as follows. First, the EHS system used is described, Second, the structure of the PFC used and the ASPR conditions of the augmented plant are given. Third, the selection criterion of the PFC parameters is proposed. Fourth, the online estimation algorithm of the natural frequency of the EHS system is presented. Fifth, the simulation and experimental results are given. Finally, the conclusion is described.

\section{CONTRL SYSTEM HARDWEAR}

In this paper, the position control of an EHS system is treated. Figure 1 shows a schematic of the EHS system for position control. The stroke, the internal diameter and the piston rod diameter of the hydraulic cylinder are $0.2 \mathrm{~m}, 0.032 \mathrm{~m}$ and $0.018 \mathrm{~m}$, respectively. The rated flow rate, pressure and current of the servo valve are $7.5 \times 10^{-4} \mathrm{~m}^{3} / \mathrm{s}, 14 \mathrm{MPa}$ and $0.03 \mathrm{~A}$, respectively. The reference input, $r$, which is supplied by a function generator, and the outputs of a potentiometer and pressure sensors are read into a computer through a 12 bit analogue-to-digital (A/D) converter. The control input is calculated by the computer according to a control scheme and supplied to a servo-amplifier through a 12 bit digital-to-analogue (D/A) converter.

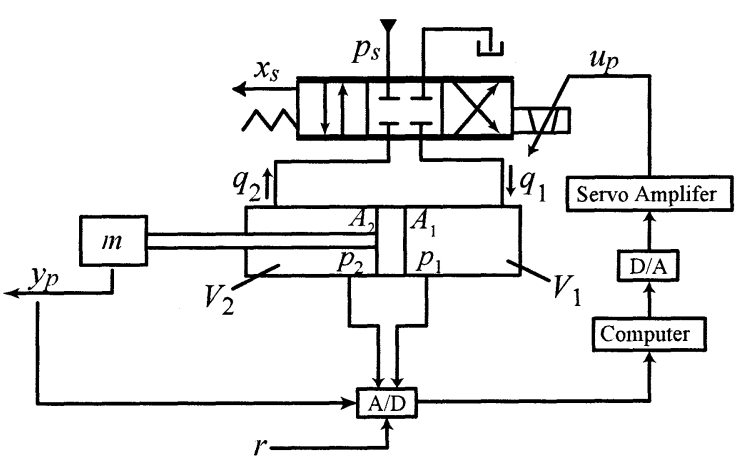

Figure 1 Schematic of position control EHS system

\section{PFC AND ASPR CONDITION}

By approximating the asymmetric cylinder as a symmetric one and by neglecting the dynamics of the servo valve, the transfer function of the EHS system can be given by

$$
G_{p}(s)=\frac{Y_{p}(s)}{U_{p}(s)}=\frac{K \omega_{n}^{2}}{s\left(s^{2}+2 \zeta \omega_{n}+\omega_{n}^{2}\right)}
$$

where $Y_{p}$ and $U_{p}$ are the Laplace transforms of the plant output (position), $y_{p}$, and the control input (servo current), $u_{p}$, respectively.

A PFC is added to the plant as shown in Fig.2. The transfer function of the PFC can be given by the following equation [4]:

$$
G_{p f c}(s)=\frac{\beta(s+11 \alpha)}{(s+\alpha)^{2}}
$$

The augmented plant is given by $G_{a}(s)=G_{p}(s)+$ 
$G_{p f c}(s)$. The ASPR conditions for an SISO system are given by the following [5]:

C1) The relative degree is 0 or 1 .

C2) The coefficient of the highest-order term in the numerator polynomial is positive.

C3) The system is minimum phase.

The above conditions $\mathrm{C} 1$ and $\mathrm{C} 2$ can be easily satisfied by selecting the PFC as given by Eq.(2) with $\beta>0$. By applying the Hurwitz's stability criterion to the numerator polynomial of the augmented plant, $G_{a}(s)$, it can be seen that the condition $\mathrm{C} 3$ holds if the PFC parameters are selected to satisfy the following inequality:

$$
\begin{aligned}
& -121 \frac{\beta}{K}\left(\frac{\alpha}{\omega_{n}}\right)^{3}+\left(2662 \frac{\beta}{K}+440\right) \zeta \frac{\beta}{K}\left(\frac{\alpha}{\omega_{n}}\right)^{2} \\
& +\left[484 \zeta^{2}\left(\frac{\beta}{K}\right)^{2}+84 \zeta^{2} \frac{\beta}{K}+99 \frac{\beta}{K}+18\right] \frac{\alpha}{\omega_{n}} \\
& +22 \zeta\left(\frac{\beta}{K}\right)^{2}+26 \zeta \frac{\beta}{K}+4 \zeta>0
\end{aligned}
$$

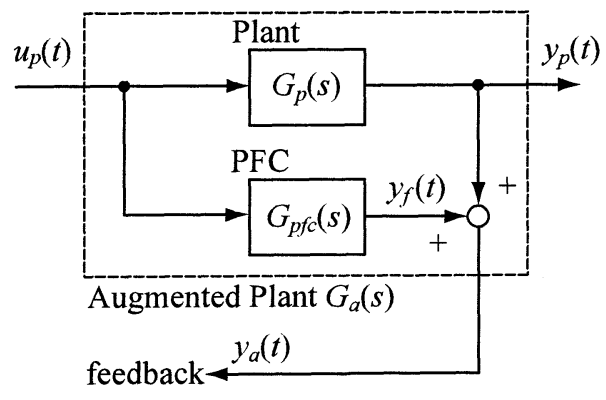

Figure 2 Augmented plant

Equation (3) shows that whether the augmented plant is ASPR or not is determined by three dimensionless parameters: $\alpha / \omega_{n}, \beta / K$ and $\zeta$. Substituting variable values into the three dimensionless parameters and calculating Eq.(3) yield the boundary surface between the ASPR and non-ASPR regions as shown in Fig.3. As can be seen from Fig.3, taking into account the fact that the value of $\zeta$ is seldom less than 0.1 for EHS systems, the augmented plant becomes ASPR when the value of $\alpha / \omega_{n}$ is in the range $0<\alpha / \omega_{n} \leq 2$. In addition, the ASPR region widens with increasing $\zeta$, and the value of $\beta / K$ does not have a great influence on the ASPR condition. Therefore, if the value of $\alpha / \omega_{n}$ can be kept in the range $0<\alpha / \omega_{n} \leq 2$ even under a large variation of $\omega_{n}$, it is possible for the augmented plant to be ASPR.

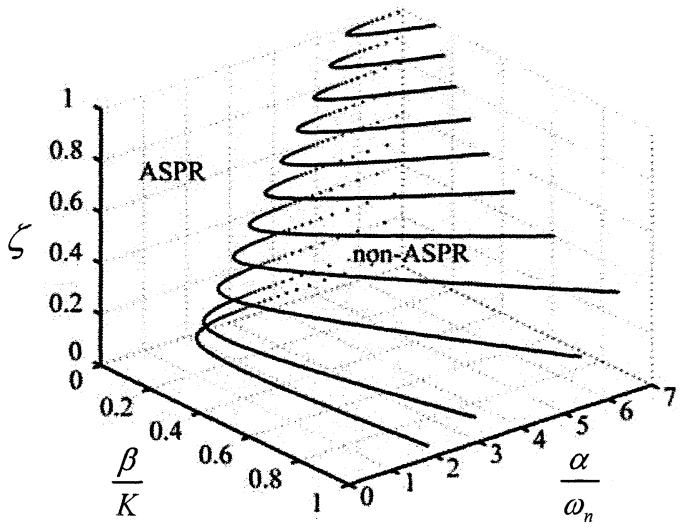

Figure 3 Boundary surface between ASPR and non-ASPR regions

\section{SELECTION CRITERION OF PFC PARAMETERS}

Even if the augmented plant is ASPR, the response of the plant may be oscillatory or slow. In order to find out the optimal values of $\alpha / \omega_{n}$ and $\beta / K$, the tracking performance was examined in simulation using the feedback system shown in Fig.4 under the variable conditions of the plant and PFC parameters. The simulation was conducted using MATLAB/Simulink.

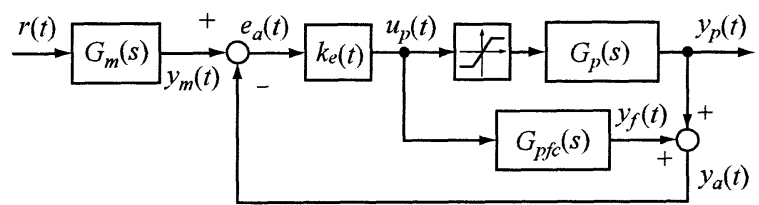

Figure 4 Evaluation system of tracking performance

A rectangular reference input whose value and frequency are $0.10 \pm 0.05 \mathrm{~m}$ and $0.3 \mathrm{~Hz}$ was used. The tracking performance was evaluated by the following function:

$$
\begin{aligned}
& J_{e}=\int_{0}^{t_{f}}\left|e_{y}(t)\right| d t \\
& e_{y}(t)=\left\{\begin{array}{cc}
y_{p}(t)-y_{m}(t) & \left|y_{p}(t)-y_{m}(t)\right|>\varepsilon \\
0 & \left|y_{p}(t)-y_{m}(t)\right| \leq \varepsilon
\end{array}\right. \\
& (\varepsilon=0.0002 \mathrm{~m})
\end{aligned}
$$

where $t_{f}$ stands for half of the period of the rectangular input. 
Figures 5 and 6 show examples of the simulation results Though not clearly seen from Figs.5 and 6, the simulations have shown the following:

R1) The range of $\alpha / \omega_{n}$ where the evaluation function becomes minimum is hardly changed by the values of the plant parameters and $\beta$, and is $1 \leq \alpha / \omega_{n} \leq 3$.

R2) The range of $\beta / K$ where the evaluation function becomes minimum is affected by the values of $\zeta$ and $\alpha$ and hardly affected by the other parameters.

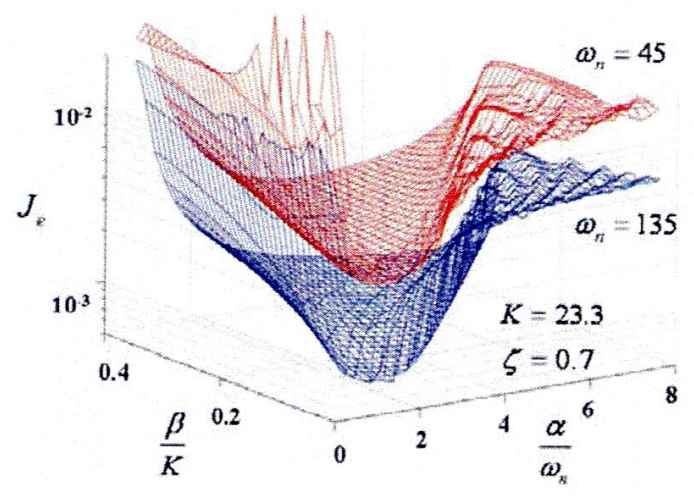

Figure $5 J_{e}$ vs. dimensionless PFC parameters (effect of $\omega_{n}$ )

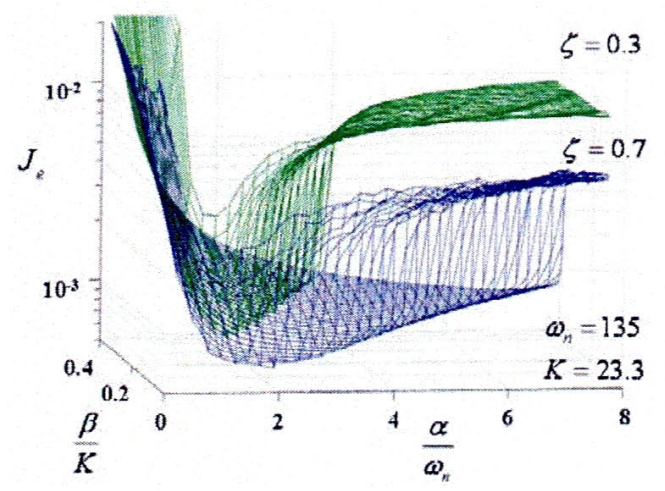

Figure $6 J_{e}$ vs. dimensionless PFC parameters (effect of $\zeta$ )

Taking these results and the results regarding the ASPR conditions given in the previous section, a method to determine the PFC parameters is proposed as follows:

P1) For the parameter $\alpha$, an arbitrary value in the range of $1 \leq \alpha / \omega_{n} \leq 2$ is selected, e.g., $\alpha=1.5 \omega_{n}$. In the control scheme proposed in this paper, the value of $\alpha$ is varied according to the variation of $\omega_{n}$.

P2) For the parameter $\beta$, a constant value is used. The value is determined by trial and error in order that a good response is obtained under the condition of the maximum $K$ and the minimum $\zeta$.

\section{ESTIMATION OF NATURAL FREQUENCY}

A theoretical expression of the natural angular frequency of the EHS system shown in Fig. 1 can be give by

$$
\omega_{n}=\frac{A_{1}+A_{2}}{2} \sqrt{\frac{B_{e}}{m}\left(\frac{1}{V_{1}}+\frac{1}{V_{2}}\right)}
$$

The values of the piston areas are known and those of the volumes in the cylinder can be known by measuring the piston position. Therefore, if the values of the load mass and effective bulk modulus of fluid can be obtained, the value of the natural frequency can be calculated. In this paper, the values of the mass and effective bulk modulus are estimated online separately using the equation of motion and equation of continuity, respectively.

The equation of motion of the system shown in Fig.1 is written by

$$
m \frac{d v}{d t}=p_{1} A_{1}-p_{2} A_{2}-F_{L}
$$

Multiplying both sides of Eq.(7) by $1 /\left(s+\lambda_{l}\right)$ (a firstorder lowpass filter) and $s /\left(s+\lambda_{h}\right)$ (a first-order highpass filter, $\lambda_{h}<<\lambda_{l}$ ) yields

$$
m\left(v_{h}-\lambda_{l} v^{\prime}\right)=A_{1} p_{1}^{\prime}-A_{2} p_{2}^{\prime}
$$

where

$$
\begin{aligned}
& v_{h}=\frac{s}{s+\lambda_{h}} v, \quad v^{\prime}=\frac{s}{\left(s+\lambda_{h}\right)\left(s+\lambda_{l}\right)} v \\
& p_{1}^{\prime}=\frac{s}{\left(s+\lambda_{h}\right)\left(s+\lambda_{l}\right)} p_{1}, \quad p_{2}^{\prime}=\frac{s}{\left(s+\lambda_{h}\right)\left(s+\lambda_{l}\right)} p_{2}
\end{aligned}
$$

In the derivation of Eq.(8), an assumption $s F_{L}=\dot{F}_{L} \approx 0$ was introduced. The velocity, $v$, is estimated by a Kalman filter and the pressures, $p_{1}$ and $p_{2}$, are measured by pressure sensors.

By letting the estimate of $m$ be $\hat{m}$, the prediction error $\varepsilon_{m}$ is defined as

$$
\begin{aligned}
& \varepsilon_{m}=\hat{m} \varphi_{m}-\left(A_{1} p_{1}^{\prime}-A_{2} p_{2}^{\prime}\right) \\
& \varphi_{m}=v_{h}-\lambda_{l} v^{\prime}
\end{aligned}
$$

Using the weighted least-squares method, a parameter update law is derived, which is given in discrete form 
by

$$
\begin{aligned}
& \hat{m}(k)=\hat{m}(k-1)-\frac{\gamma_{m}(k-1) \varphi_{m}(k)}{\lambda+\varphi_{m}^{T}(k) \gamma_{m}(k-1) \varphi_{m}(k)} \varepsilon_{m}(k) \\
& \gamma_{m}(k)=\frac{1}{\lambda}\left[\gamma_{m}(k-1)-\frac{\gamma_{m}^{2}(k-1) \varphi_{m}^{2}(k)}{\lambda+\varphi_{m}^{2}(k) \gamma_{m}(k-1)}\right]
\end{aligned}
$$

where $\lambda$ is the weight and

$$
\hat{m}(0)=\hat{m}_{0}, \gamma_{m}(0)=\gamma_{m 0}>0
$$

The equations of continuity are described by

$$
\begin{aligned}
& \frac{V_{1}}{B_{e}} \frac{d p_{1}}{d t}=q_{1}-A_{1} v \\
& \frac{V_{2}}{B_{e}} \frac{d p_{2}}{d t}=A_{2} v-q_{2}
\end{aligned}
$$

Likewise, multiplying both sides of Eqs.(14), (15) by $1 /\left(s+\lambda_{l}\right)$ and substituting the estimate, $\hat{B}_{e}$, for $B_{e}$ yield the following prediction error:

$$
\begin{gathered}
\varepsilon_{B_{e}}=\hat{B}_{e}^{-1} \varphi_{B_{e}}-\left[q_{1 l}+q_{2 l}-\left(A_{1}+A_{2}\right) v_{l}\right] \\
\varphi_{B_{e}}=V_{1}\left(p_{1}-\lambda_{l} p_{1 l}\right)-V_{2}\left(p_{2}-\lambda_{l} p_{2 l}\right)
\end{gathered}
$$

where the subscript $l$ indicates the lowpass filtered variables. The values of the flow rates, $q_{1}$ and $q_{2}$, are calculated using the nonlinear flow rate characteristic equations of the servo valve; the spool displacement is estimated by assuming the spool dynamics as the first-order system. A parameter update law for $\hat{B}_{e}$ similar to Eqs.(12),(13) is obtained.

By substituting $\hat{m}$ and $\hat{B}_{e}$ for $m$ and $B_{e}$ in Eq.(6), the estimate, $\hat{\omega}_{n}$, of $\omega_{n}$ can be calculated.

\section{RESULTS}

In this investigation, in order to make clear the effect of the online tuning of the PFC parameter, a simple proportional control with a variable gain shown in Fig.7 was used. The block written as Estimator includes the algorithm to estimate the natural frequency. The PFC parameter $\alpha$ was adaptively varied according to the variation of the value of the natural frequency; the adaptive rule was $\alpha=2 \hat{\omega}_{n}$. Another parameter $\beta$ was kept at a constant value determined offline in advance. The proportional gain was generated according to the following adaptive rule with a constant weight, $\gamma$ :

$$
\begin{aligned}
& k_{e}(t)=k_{e 0}+\gamma\left|e_{a}(t)\right| \\
& e_{a}=y_{m}-y_{a}
\end{aligned}
$$

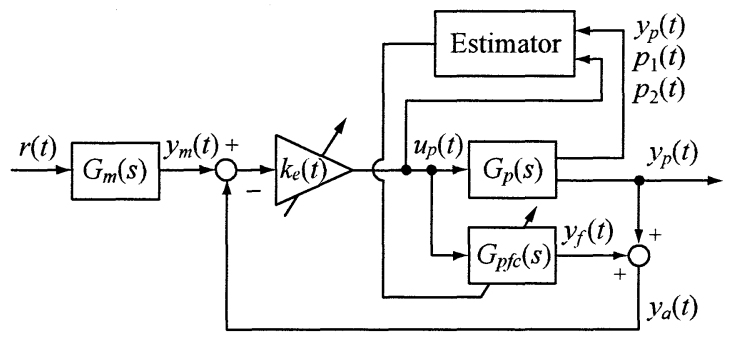

Figure 7 Block diagram of control system
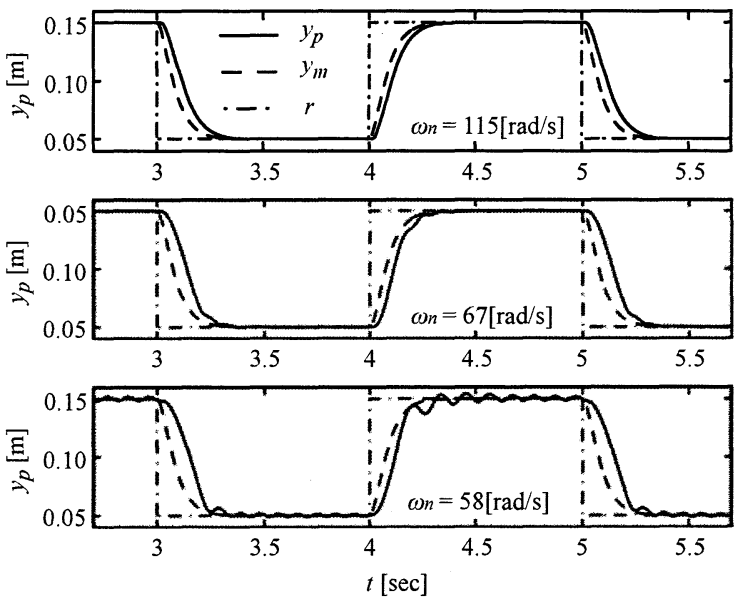

Figure 8 Simulation results under constant $\alpha$ (conditions: $\alpha=2 \hat{\omega}_{n}, \hat{\omega}_{n}=115 \mathrm{rad} / \mathrm{s}$ (fixed) $\beta=0.52$ )

Figure 8 shows simulation results obtained under the condition that the PFC parameter $\alpha$ was kept at a certain value. When the natural frequency of the EHS system is identical to the design value (115 rad/s, upper figure), the response is good. In case where the natural frequency is decreased to $67 \mathrm{rad} / \mathrm{s}$ (middle figure) due to the increase of the load mass and/or the decrease of the stiffness of the fluid, the response is deteriorated to some degree but not bad. In this case, the real value of $\alpha / \omega_{n}$ is 3.4. However, a further decrease in the natural frequency to $58 \mathrm{rad} / \mathrm{s}$, which leads to the increase in $\alpha / \omega_{n}$ to 4.0 , causes an oscillatory motion (lower figure). As can be seen from Fig.8, the control performance may be deteriorated under a constant $\alpha$ when the variation of the plant parameters is large.

Figure 9 shows a simulation result obtained when the estimation of the natural frequency and the adaptation of the parameter $\alpha$ were made. Before $t=5 \mathrm{sec}$, the 
parameter $\alpha$ is fixed at a constant value $(230 \mathrm{rad} / \mathrm{s})$ designed using the value of the natural frequency initially estimated $(115 \mathrm{rad} / \mathrm{s})$. In the same manner as the lower figure in Fig.8, an oscillatory motion takes place. At the instant the estimation of the natural frequency and the adaptation of $\alpha$ accompanying it start at $t=5 \mathrm{sec}$, the response becomes smooth. This is because, as can be seen from the lower figure in Fig.9, the natural frequency can be quickly estimated in a relatively good accuracy. The usefulness of the control scheme proposed has been demonstrated by simulation.
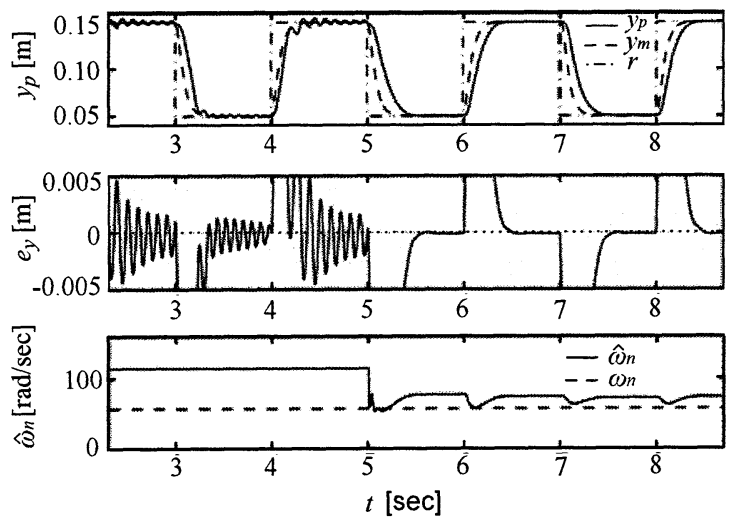

Figure 9 Simulation result under adaptive $\alpha$ (conditions: $\alpha=2 \hat{\omega}_{n} . \hat{\omega}_{n}$ is estimated online. $\beta=0.52$. Initial value of $\omega_{n}$ used for PFC design is $115 \mathrm{rad} / \mathrm{s}$. Real value of $\omega_{n}=58 \mathrm{rad} / \mathrm{s}$. Estimation of $\omega_{n}$ and adaptation of $\alpha$ start at $t=5 \mathrm{sec}$.)

An experimental result is shown in Fig.10. The experimental conditions are almost the same as those in the simulation shown in Fig.9, except for the initial value of the natural frequency of the EHS system. The dashed and dash-dot lines in the lower figure in Fig.10 show the values of the natural frequency estimated offline by two methods: the least-squares method using the ARX model and the self-excited vibration method [6]. The results obtained by the two estimation methods are roughly identical and the mean value $(60 \mathrm{rad} / \mathrm{s})$ of the two can be regarded as the true natural frequency. The response is very steep in the vicinity of the reference input ( $\left.y_{p}=r=0.15 \mathrm{~m}\right)$ before the adaptation of $\alpha$ begins at $t=5 \mathrm{sec}$. However, after the beginning of the adaptation of $\alpha$ at $t=5 \mathrm{sec}$, the response becomes smooth near both at $y_{p}=r=0.15 \mathrm{~m}$ and at $0.05 \mathrm{~m}$. The estimation of the natural frequency is made relatively quickly and accurately, though not so fast compared to the simulation shown in Fig.9. The usefulness of the control scheme proposed has been demonstrated also by experiment.
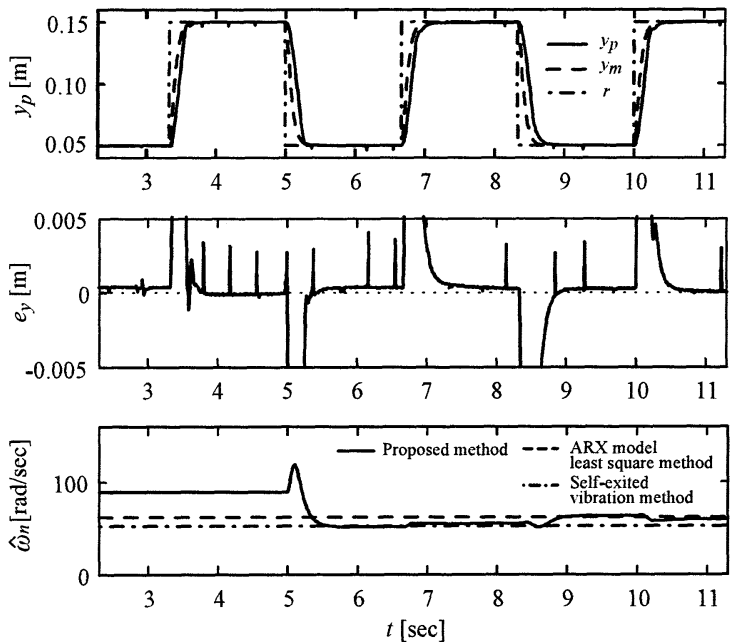

Figure 10 Experimental result under adaptive $\alpha$ (conditions: $\alpha=2 \hat{\omega}_{n} . \hat{\omega}_{n}$ is estimated online. $\beta=4.2$. Initial value of $\omega_{n}$ used for PFC design is $90 \mathrm{rad} / \mathrm{s}$. Real value of $\omega_{n} \approx 60 \mathrm{rad} / \mathrm{s}$. Estimation of $\omega_{n}$ and adaptation of $\alpha$ start at $t=5 \mathrm{sec}$.)

\section{CONCLUSION}

In this paper, a criterion to determine the values of the PFC parameters, an online estimation method of the natural frequency of an EHS system and an adaptive, robust control scheme with the PFC have been proposed. It has been shown that the natural angular frequency can be estimated online in a relatively good accuracy by the method proposed, and that the control performance of the EHS system can be enhanced by adaptively changing a PFC parameter according to the variation of the natural frequency.

\section{REFERENCES}

1. Kaufman, H., Barkana, I. and Sobel, K., Direct Control Algorithms : Theory and Applications, Springer, 1997.

2. Iwai, Z, Egarashi, T, Hino, M., Takahashi, M. and Kyoizumi, K., Trans. Japan Soc. Mech. Engrs. (Ser. C), 1995, 61-590, pp.3991-3998.

3. Tsukamoto, N. and Yokota, S., Trans. Japan Fluid Power System Soc., 2003, 34-6, pp.126-133.

4. Iwai, Z. and Mizumoto, I., Int. J. Control, 1992, 55-6, pp.1453-1470.

5. Zeheb, H., IEEE Trans., 1986, AC-31-11, pp.10551057.

6. Nishiumi, T., Ichiyanagi, T., Katoh, H. and Konami, S., Trans. Japan Fluid Power System Soc., 2005, 36-1, pp.1-7. 